\title{
Neuroblastoma in Vietnam: A retrospective analysis of $M Y C N$ status and clinical features to inform prognosis and improve outcome
}

\author{
Chi-Bao Bui ${ }^{1,4 * *}$, Uyen HT Nguyen ${ }^{1 \#}$, Diem KD Truong ${ }^{4}$, Niem VT Vo ${ }^{4}$, Diem-My Vu ${ }^{6}$, Uyen DP Nguyen ${ }^{1}, B_{a n}$ T Ho ${ }^{5}$, Van D Nguyen ${ }^{3}$, \\ Dinh QTruong ${ }^{2}$ and Khai D Truong ${ }^{5 *}$ \\ ${ }^{1}$ Department of Molecular Genetics, City Children's Hospital, Ho Chi Minh City, Vietnam \\ ${ }^{2}$ Department of Surgery, City Children's Hospital, Ho Chi Minh City, Vietnam \\ ${ }^{3}$ Department of Hematology and Oncology, Children's Hospital 2, Ho Chi Minh City, Vietnam \\ ${ }^{4}$ Center for Molecular Biomedicine, University of Medicine and Pharmacy at Ho Chi Minh City, Vietnam \\ ${ }^{5}$ Department of Pediatric Surgery, University of Medicine and Pharmacy at Ho Chi Minh City, Vietnam \\ ${ }^{6}$ Molecular Medicine, International Centre for Genetic Engineering and Biotechnology, AREA Science Park, 34149 Trieste, Italy \\ \#These authors contributed equally to this work
}

\begin{abstract}
Background: Neuroblastoma is the most common childhood cancer worldwide. While recent advances in diagnosis and treatment have resulted in dramatic improvements in patient survival, neuroblastoma recurrence and mortality rates in Vietnam are unknown. This study evaluated the survival rates of Vietnamese patients with NB and investigated the impact of clinical factors and MYCN status on prognosis.

Methods: This study analysed survival rates, clinical factors and MYCN status of NB patients across three hospitals in Ho Chi Minh City. Kaplan-Meier and multivariable Cox proportional hazards models were used to describe Survival rates and determine hazard ratios. Patient samples were analysed retrospectively for MYCN amplification by fluorescence in-situ hybridization (FISH) and quantitative polymerase chain reaction (qPCR).

Results: 5 -year overall survival (OS) was $39.78 \% \pm 4.31 \%$ and event-free survival (EFS) was $56.63 \% \pm 5.47 \%$ in our cohort, and significantly lower than OS and EFS rates in Europe, USA, Japan, China and Thailand. $M Y C N$-amplified or Stage III and IV NB patients showed significantly decreased survival time in comparison to those without $M Y C N$ amplification $(\log$ rank $P=0.000)$ or diagnosed at stage I, II and IVs $(\log$ rank $P=0.000)$. Furthermore, patients older than 18 months or at stage III and IV had a significantly higher probability of recurrence $(P=0.038$ and $P=0.000$, respectively).
\end{abstract}

Conclusions: This study demonstrates poor outcome for NB patients in Vietnam. Furthermore, prognosis is significantly worsened by their $M Y C N$ status; NB stage and age at diagnosis.

\section{Introduction}

Neuroblastoma (NB) is the most common cause of solid cancerrelated death in children, with 10.2 cases per million under the age of 15 [1]. In the USA and Europe, NB is responsible for 8-10\% of all child cancers [2-5]. Sadly the survival rate of NB patients differs based on the country's income level with risk of death far higher in low-middle income countries (LMIC) than in high-income countries (HIC) [6]. The reasons underlying such disparity are complex but include late diagnosis, inaccurate staging, poor risk stratification, suboptimal therapy, and lack of education in LMIC's [6]. Vietnam, an LMIC country, experiences many of these issues however, due to insufficient access to patient clinical records there are no reports outlining NB survival rates or prognostic factors.

The MYCN gene, encodes an oncogenic transcription factor and is widely reported to play an important role in NB differentiation $[7,8]$. MYCN expression decreases during the NB differentiation process and high levels of $M Y C N$ are associated with decreased cancer differentiation and a neoplastic phenotype [7]. MYCN gene amplification is perhaps the best characterised molecular biomarker of risk in NB and it has been reported that approximately $20 \%$ of NB cases demonstrate $M Y C N$ amplification [8,9]. Therefore, it is necessary to estimate the NB survival rate overall as well as stratifying patients based on $M Y C N$-amplification status.

The objective of this study was to investigate NB survival rates and outcomes in Vietnam and evaluate how these related to clinical characteristics and $M Y C N$ amplification in order to determine optimal prognostic indicators.

*Correspondence to: Chi-Bao Bui, Center for Molecular Biomedicine, University of Medicine and Pharmacy, Ho Chi Minh City, Vietnam, E-mail: bcbao@ump.edu.vn

Khai Dinh Truong, Department of Pediatric Surgery, University of Medicine and Pharmacy at Ho Chi Minh City, Vietnam, E-mail: doctorkhai2014@gmail.com

Key words: pediatrics, surgery, neuroblastoma, MYCN, vietnam

Received: September 09, 2019; Accepted: September 26, 2019; Published: September 30, 2019 


\section{Materials and methods}

\section{Study design and patients}

Patients at the Hematology Oncology Department of Children's Hospital I, II and Oncology Hospital in Ho Chi Minh from January 2010 to December 2012 who were diagnosed with NB were chosen for this study. The primary inclusion criteria for the study was the presence of clinical records showing follow up of at least five years and including all information on clinical presentations related to gender, age, International Neuroblastoma Staging System (INSS) scoring, tumour origin, relapse or progression after first-line of treatment, firsttime of relapse, $M Y C N$ status and outcome. MYCN status was classified as non-amplification or amplification (> 10 copies) following standard guidelines as determined by fluorescence in-situ hybridization (FISH) and quantitative polymerase chain reaction (qPCR) [10-12].

\section{Statistical analysis}

SPSS software was used for data analysis. Associations between clinical factors, MYCN status and outcome were estimated using the two-tailed $\chi 2$-test or Fisher's exact test. Event-free survival (EFS) time was calculated as the time from diagnosis to first evidence of an event which included relapse, progression and death by any cause. Overall survival (OS) time was calculated as the time from diagnosis to death, or until last diagnosis. OS and EFS was analysed by Kaplan-Meier and log-rank test. Multivariable Cox regression was used to estimate the prognostic value of gender, age, INSS and MYCN status with respect to survival time after recurrence. Each factor was divided into groups for analysis: "gender" consisted of: (1) female (2) male; "age": (1) $\leq$ 18 months (2) > 18 months; "INSS" stage: (1) stage I, II and IVs and (2) stage III and IV; "tumour origin": (1) adrenal (2) abdominal (3) thoracic; "MYCN status": (1) MYCN non-amplification (2) MYCN amplification. Predictive positive and negative values (PPV and $\mathrm{NPV}$ ) of MYCN status in respect to outcome were also calculated. All $P$-values were calculated by two-sided testing and values below 0.05 were considered statistically significant.

\section{Results}

A total of 130 samples satisfied all criteria for analysis. The median time of observation was 26 months (ranging 3 to 90 months). OS rate was $39.78 \% \pm 4.31 \%$ while EFS rate was $56.63 \% \pm 5.47 \%$. 48 patients (36.92\%) were found to be recurrent, 32 patients showed metastases (24.62\%) and 89 NB-related deaths were recorded (68.46\%) (Table 1). The time from diagnosis to first relapse was in the range of 2-20 months and the median time of this range was 13 months. 76 (58.46\%) of patients were male and 54 (41.54\%) female. 96 (73.85\%) of patients were over 18 months old and $33(25.38 \%)$ were 18 months old or under (Table 1). 96 patients were at NB stage III and IV (73.85\%) versus 34 patients at stage I, II and IVs (25.38\%) (Table 1). The majority of patients had tumours of adrenal origin (106 patients, $81.54 \%$ ) while 17 patients (13.08\%) had tumours of abdominal origin and 4 patients (3.08\%) were of thoracic origin (Table 1). 33 patients showed a first time to relapse greater than or equal to 12 months $(25.38 \%)$ that was around twice that of patients with a first time to relapse less than 12 months (15 patients, 11.54\%). There was a statistically significant association between recurrence and NB disease at INSS stage III and IV $(P=0.000)$ (Table 1). An age of greater than 18 months was also associated with disease recurrence $(P=0.038)$ (Table 1$)$.

There were $89 \mathrm{NB}$-associated deaths in OS analysis and 43 events recorded in EFS analysis (Figure 1). The survival rate of children with
NB declined to around $39.78 \% \pm 4.31 \%$ for OS and EFS declined to $56.63 \% \pm 5.47 \%$ after 40 months. After 40 months both OS and EFS further decreases by a narrow margin following which there is a plateau until the end point of the study at five years follow up (Figure 1).

Patients with stage III and IV NB show significantly decreased OS $(P=0.00)$ and EFS $(P=0.00)$ (Figure 2). After 5-years follow-up, patients with stage III and IV NB had OS rate of $13.54 \% \pm 3.56 \%$ while patients with stage I, II and IVs NB had OS rate as high as $79.41 \% \pm 6.93 \%$. Similarly, patients with stage III and IV NB had EFS rates of $31.21 \% \pm$ $7.03 \%$ and patients with stage I, II and IVs NB had EFS rates of $80.99 \%$ $\pm 6.99 \%$ (Figure 2). After 5 years observation, patients with stage III and IV NB had a median OS time of 24.00 (95\%CI 20.53-27.47) months and an EFS time of 27.00 (95\%CI 24.41-29.59) months while patients with stage I, II and IVs NB showed significantly greater survival times with a median OS ( $>45$ months) and an EFS ( $>45$ months). (Supplementary Table S1; $P=0.000)$.

Although patients older than 18 months showed rates of relapse significantly higher than those aged 18 months or less, age had no statistically significant impact on the overall outcome of NB (OS: $\log$ rank $P=0.054$; EFS: log rank $P=0.98$ ) (Supplementary Figure S2). Notably, although OS rate and EFS rate was lower in male versus female NB patients, this only reached statistical significance for EFS where the median was 29.00 (95\%CI 21.98-36.02) months for males and $>45$ months for females $(P=0.045)$ (Supplementary Figure S1, Supplementary Table S1).

MYCN-amplification was detected in 23 samples, accounting for $17.69 \%$ of patients (Table 1). MYCN-amplified patients showed a significantly lower OS rate of $13.04 \% \pm 7.02 \%$ and EFS rate of $28.12 \%$ \pm 13.21 in comparison with MYCN non-amplified patients $(35.03 \%$ $\pm 4.64 \%$ and $55.02 \% \pm 6.04 \%$, respectively) after a 5 -year followup (Figure 2). The median OS and EFS of $M Y C N$-amplified patients was 18.00 (95\%CI $15.65-20.35)$ months and 19.00 (95\%CI $13.204-$ 24.80) months, respectively which was significantly lower than those of MYCN non-amplified patients $(P=0.000$ for OS and $P=0.006$ for EFS) (Supplementary Table S1). Furthermore, the NPV of MYCNamplification was high for OS (87.0\%) but low for EFS (43.5\%) (Table 2). $M Y C N$-amplification showed high specificity and sensitivity rates for the prediction of OS ( $92.7 \%$ and $85.1 \%$, respectively) (Table 2). The accuracy rate of the test is low at $44.6 \%$ for OS but higher for EFS (64.6\%) (Table 2).

MYCN status and INSS stage significantly associated with OS and EFS in both univariate and multivariate analyses (Table 3). Multivariate analysis illustrated that MYCN status predicted OS and EFS independently of gender, age and INSS stage of patients (Table 3). Gender and age variables were not significantly associated with outcome when tested individually, however, when combined with $M Y C N$-amplification variable, their interactions had a statistically significant association with OS and EFS (OS $P=0.026$ and $P=0.000$; EFS $P=0.021$ and $P=0.007$, respectively) (Table 3 ). Male patients or patients older than 18 months with $M Y C N$-amplification showed a significant association with outcome of NB compared with male patients or patients less than 18 months with $M Y C N$ non-amplified (OS $P=0.022$ and $P=0.000$; EFS $P=0.071$ and $P=0.004$, respectively) (Table 3) (Supplementary Table S2).

\section{Discussion}

This study examined the outcome of NB in Ho Chi Minh City, Vietnam and further assessed accompanying clinical/MYCN 
Table 1. Prognostic significance of clinical factors and MYCN-status in recurrence of NB patients.

\begin{tabular}{|c|c|c|c|c|c|c|c|}
\hline \multirow[t]{2}{*}{ Variable } & \multirow{2}{*}{$\begin{array}{c}\text { All patients } \\
\text { No. (\%) }\end{array}$} & \multicolumn{2}{|c|}{ Recurrence } & \multirow{2}{*}{$\begin{array}{c}\text { Death } \\
\text { No. }(\%) \\
\end{array}$} & \multirow{2}{*}{$\begin{array}{c}\text { Metastasis } \\
\text { No. (\%) }\end{array}$} & \multicolumn{2}{|c|}{ MYCN - amplified } \\
\hline & & No. $(\%)$ & $P$-value & & & No. $(\%)$ & $P$-value \\
\hline All patients & 130 & $48(36.92)$ & & $89(68.46)$ & $32(24.62)$ & $23(17.69)$ & \\
\hline \multicolumn{8}{|l|}{ Gender } \\
\hline Male & $76(58.46)$ & $26(34.21)$ & \multirow{2}{*}{0.466} & $55(42.31)$ & $20(15.39)$ & $14(18.42)$ & \multirow{2}{*}{1.000} \\
\hline Female & $54(41.54)$ & $22(40.74)$ & & $34(26.15)$ & $12(9.23)$ & $9(16.67)$ & \\
\hline \multicolumn{8}{|l|}{ Age } \\
\hline$\leq 18$ months & $33(25.38)$ & $7(21.21)$ & \multirow{3}{*}{0.038} & $16(12.31)$ & $2(1.54)$ & $7(21.21)$ & \multirow{3}{*}{0.601} \\
\hline$>18$ months & $96(73.85)$ & $40(41.67)$ & & $72(55.39)$ & $30(23.08)$ & $16(16.67)$ & \\
\hline ND & $1(0.77)$ & & & 1 & 0 & & \\
\hline \multicolumn{8}{|c|}{ INSS tumor stage - group } \\
\hline Stage $1,2,4 \mathrm{~s}$ & $34(26.15)$ & $2(5.88)$ & \multirow[b]{2}{*}{0.000} & $7(5.39)$ & $3(2.31)$ & $5(14.71)$ & \multirow{2}{*}{0.794} \\
\hline Stage 3,4 & $96(73.85)$ & $46(47.92)$ & & $82(63.08)$ & $29(22.31)$ & $18(18.75)$ & \\
\hline \multicolumn{8}{|l|}{ Sites of tumor } \\
\hline adrenal & $106(81.54)$ & $42(39.62)$ & & $76(58.46)$ & $27(20.77)$ & $18(16.98)$ & \\
\hline abdominal & $17(13.08)$ & $5(29.41)$ & & $8(6.154)$ & $4(3.08)$ & $5(29.41)$ & \\
\hline thoracic & $4(3.08)$ & $1(25.00)$ & & $3(2.31)$ & $1(0.77)$ & 0 & \\
\hline ND & $3(2.31)$ & 0 & & $2(1.54)$ & 0 & 0 & \\
\hline \multicolumn{8}{|l|}{ Time to first relapse } \\
\hline$<12$ months & $15(11.54)$ & & & $15(100)$ & $6(40.00)$ & $4(26.67)$ & \\
\hline$\geq 12$ months & $33(25.38)$ & & & $33(100)$ & $15(45.5)$ & $6(18.18)$ & \\
\hline $\begin{array}{l}\text { MYCN-amplification } \\
\text { Yes } \\
\text { No }\end{array}$ & $\begin{array}{c}23(17.69) \\
107(82.31)\end{array}$ & $\begin{array}{c}11(8.46) \\
37(28.46)\end{array}$ & 0.484 & $\begin{array}{l}20(15.39) \\
69(53.08)\end{array}$ & $\begin{array}{c}6(4.62) \\
26.00(20.00)\end{array}$ & & \\
\hline
\end{tabular}

NB: Neuroblastoma; INSS: International Neuroblastoma Staging System; ND: Not determined; $P$-value less than 0.05 considered significant.

Table 2. Predictive value analysis for sensitivity, specificity and accuracy rate for $M Y C N$ amplification status.

\begin{tabular}{|c|c|c|c|c|c|c|c|c|}
\hline & \multicolumn{4}{|c|}{ Overall Survival } & \multicolumn{4}{|c|}{ Event-free survival } \\
\hline & Alive & Death & PPV & NPV & No event & Event & PPV & NPV \\
\hline $\begin{array}{l}M Y C N \\
\text { non-amplification }\end{array}$ & $38(92.7 \%)$ & $69(77.5 \%)$ & \multirow{2}{*}{$87.0 \%$} & \multirow[t]{2}{*}{$35.5 \%$} & $74(85.1 \%)$ & $33(76.7 \%)$ & \multirow[t]{2}{*}{$43.5 \%$} & \multirow[t]{2}{*}{$69.2 \%$} \\
\hline \multirow[t]{2}{*}{$M Y C N$-amplification } & $3(7.3 \%)$ & $20(22.5 \%)$ & & & $13(14.9 \%)$ & $10(23.3 \%)$ & & \\
\hline & Sensitivity & Specificity & \multicolumn{2}{|c|}{ Accuracy } & Sensitivity & Specificity & \multicolumn{2}{|c|}{ Accuracy } \\
\hline$M Y C N$-amplification & $22.5 \%$ & $92.7 \%$ & \multicolumn{2}{|c|}{$44.6 \%$} & $23.3 \%$ & $85.1 \%$ & \multicolumn{2}{|c|}{$64.6 \%$} \\
\hline
\end{tabular}

PPV: Positive predictive value; NPV: Negative predictive value.

Table 3. Cox regression analysis

\begin{tabular}{|c|c|c|c|c|c|c|}
\hline Variables & $\mathrm{HR}$ and $95 \% \mathrm{CI}$ & p-value & Variables & $\mathrm{HR}$ and $95 \% \mathrm{CI}$ & p-value & $\begin{array}{c}\text { Interaction } \\
\text { p-value }\end{array}$ \\
\hline \multicolumn{7}{|l|}{ Overall survival } \\
\hline \multicolumn{3}{|l|}{ Univariate } & \multicolumn{4}{|c|}{ Multivariate } \\
\hline$M Y C N$-amplification & $2.383(1.433-3.962)$ & 0.001 & & & & \\
\hline Gender (male) & $1.132(0.738-1.738)$ & 0.569 & $\begin{array}{c}M Y C N \text {-amplification } \\
\text { Gender } \\
\text { (male) }\end{array}$ & $\begin{array}{l}2.367(1.423-3.939) \\
1.109(0.712-1.680)\end{array}$ & $\begin{array}{l}0.001 \\
0.683\end{array}$ & 0.026 \\
\hline Age $(>18)$ & $1.680(0.976-2.890)$ & 0.061 & $\begin{array}{c}M Y C N \\
\text { Age }(>18)\end{array}$ & $\begin{array}{l}2.674(1.587-4.508) \\
1.920(1.105-3.336)\end{array}$ & $\begin{array}{l}0.000 \\
0.021\end{array}$ & 0.000 \\
\hline Stage (stage-group (2)) & $7.804(3.579-17.015)$ & 0.000 & $\begin{array}{c}M Y C N \\
\text { Stage (stage-group (2)) }\end{array}$ & $\begin{array}{c}2.330(1.397-3.887) \\
7.773(3.561-16.968)\end{array}$ & $\begin{array}{l}0.001 \\
0.000\end{array}$ & 0.000 \\
\hline \multicolumn{7}{|l|}{ Event-free survival } \\
\hline \multicolumn{3}{|l|}{ Univariate } & \multicolumn{4}{|c|}{ Multivariate } \\
\hline$M Y C N$-amplification & $2.658(1.288-5.487)$ & 0.008 & & & & \\
\hline Gender (male) & $1.943(0.996-3.793)$ & 0.052 & $\begin{array}{l}M Y C N \\
\text { Gender } \\
\text { (male) }\end{array}$ & $\begin{array}{l}2.520(1.221-5.202) \\
1.859(0.951-3.636)\end{array}$ & $\begin{array}{l}0.012 \\
0.070\end{array}$ & 0.021 \\
\hline Age $(>18)$ & $1.609(0.770-3.361)$ & 0.206 & $\begin{array}{c}M Y C N \\
\text { Age }(>18)\end{array}$ & $\begin{array}{l}3.390(1.549-7.418) \\
2.101(0.963-4.584)\end{array}$ & $\begin{array}{l}0.002 \\
0.062\end{array}$ & 0.007 \\
\hline Stage (Stage-group (2)) & $4.935(2.057-11.844)$ & 0.000 & $\begin{array}{c}M Y C N \\
\text { Stage (stage-group (2)) }\end{array}$ & $\begin{array}{c}2.856(1.369-5.960) \\
5.108(2.123-12.290)\end{array}$ & $\begin{array}{c}0.005 \\
<0.001\end{array}$ & 0.009 \\
\hline
\end{tabular}

INSS: International Neuroblastoma Staging System; HR: Hazard Ratio; CI = Confidence Interval. P-values are two-sided. $P$-value less than 0.05 considered significant. Interaction P-values gained from Cox regression model: 

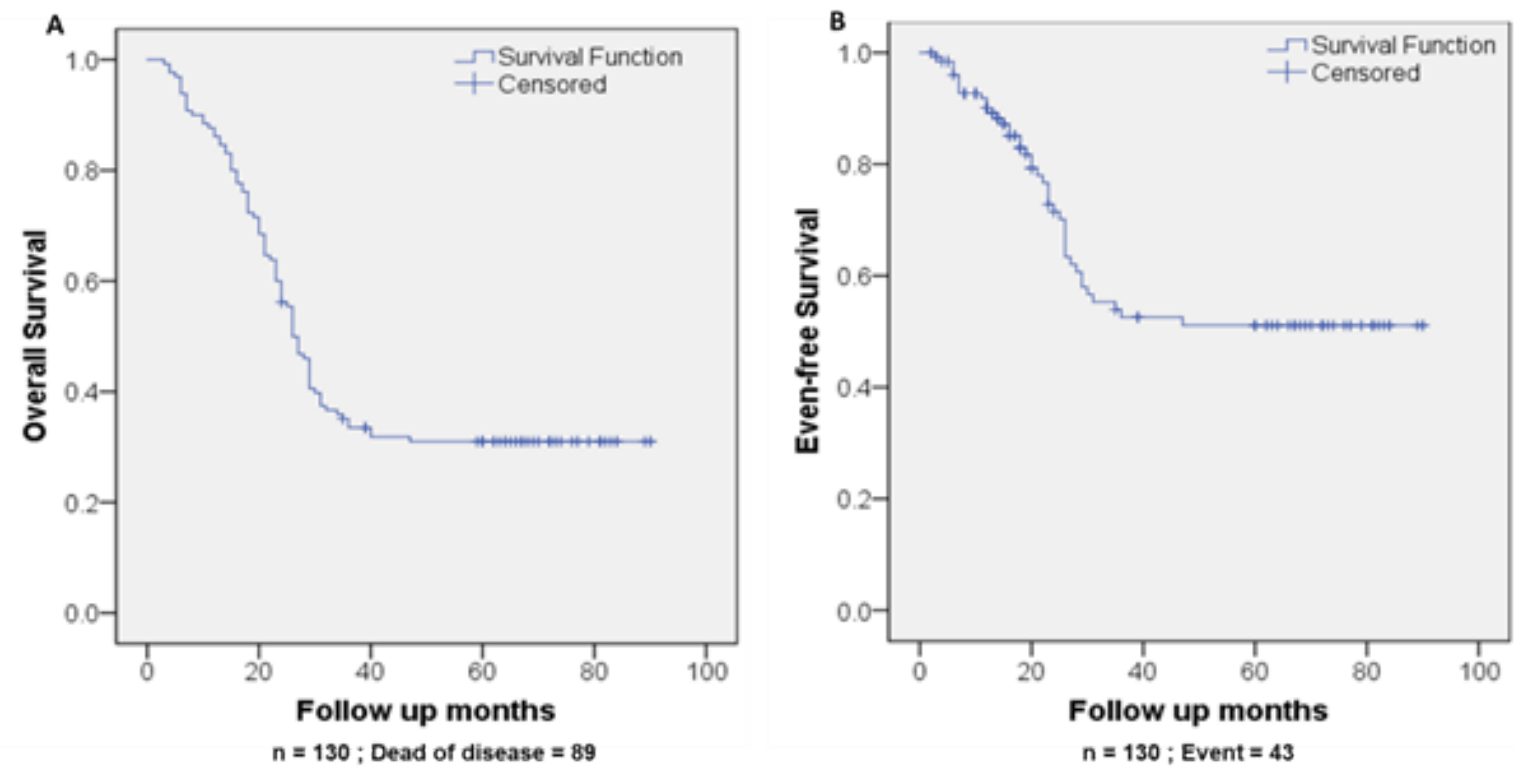

Figure 1. Survival of NB patients in 5-year observation. Kaplan-Meier curves of OS (A) and EFS (B) ( $n=130)$. $M Y C N$-amplification $+\mathrm{Co}$-factor $+M Y C N$-amplification* $\mathrm{Co}$-factor.
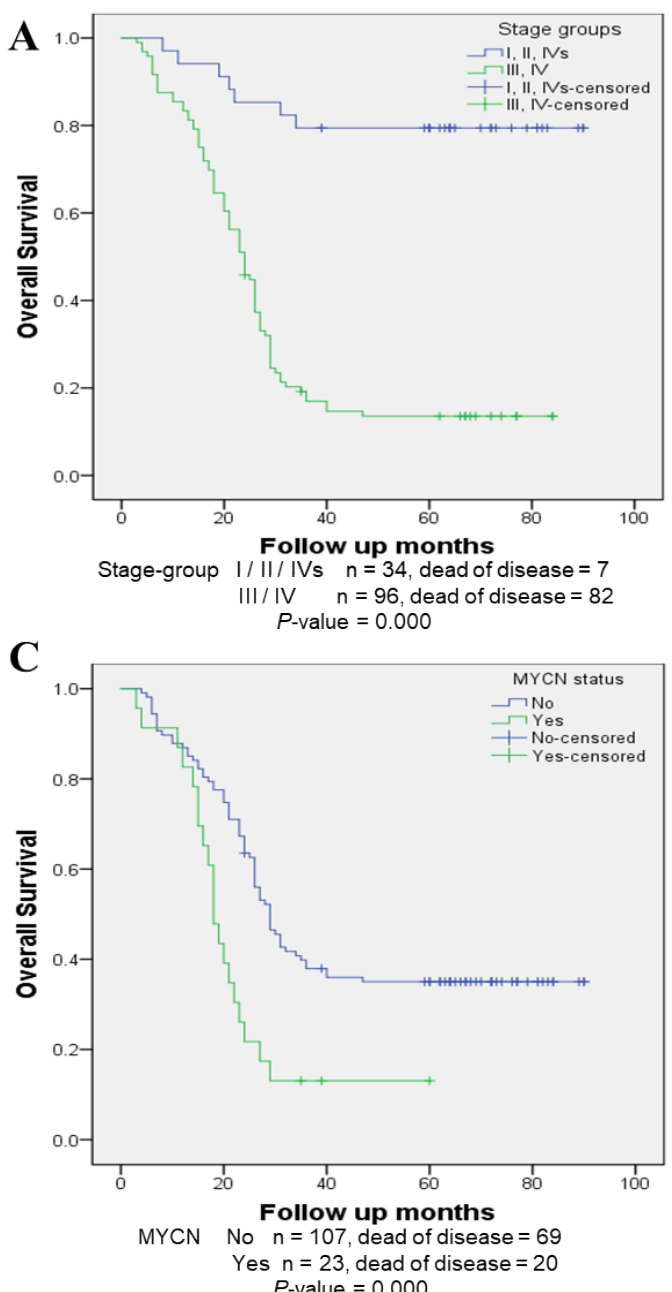

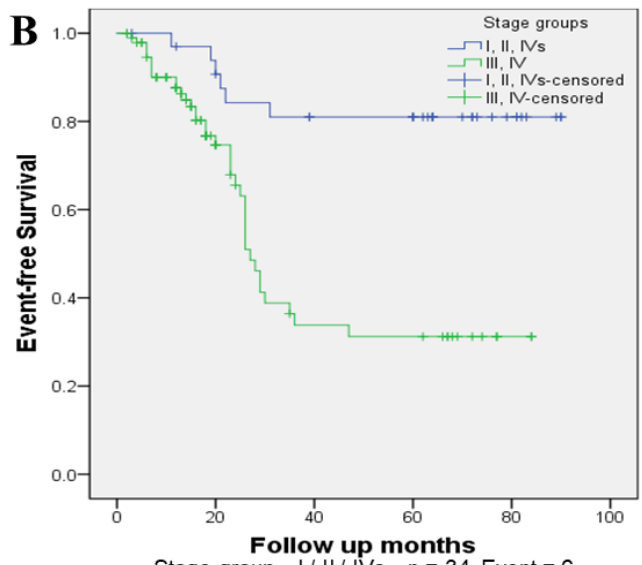

Stage-group I/II/IVs $\mathrm{n}=34$, Event $=6$

III/ IV $n=0.000$

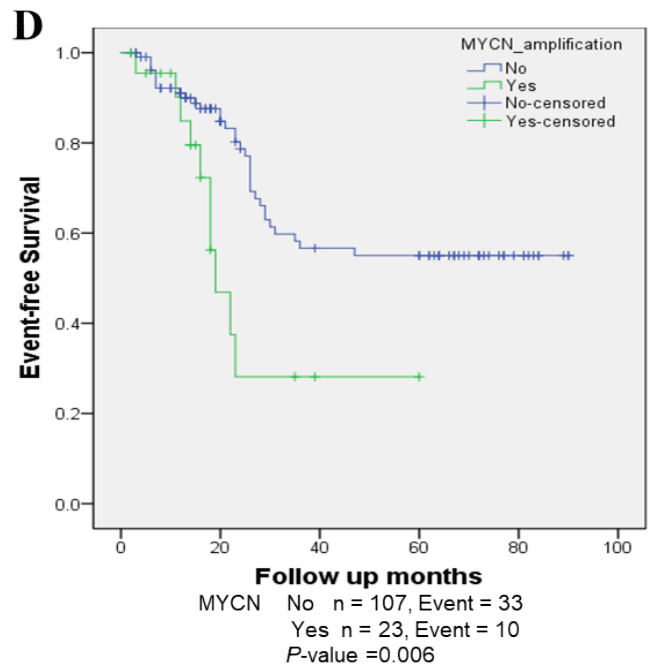

Figure 2. Survival analysis by $M Y C N$-amplification and NB stage (A) OS (log-rank test, $P=0.00$ ) and (B) EFS (log-rank test, $\mathrm{P}=0.00)$ of patients at stage III and IV; (C) OS (log-rank test, $P=0.00$ ) and (D) EFS (log-rank test, $P=0.006$ ) of NB samples according to $M Y C N$ status. 
information for potential prognostic indicators in order to fill existing knowledge gaps. In 2010-2012, there were 130 cases of NB in our cohort with 5 -year survival rates $(39.78 \% \pm 4.31 \%$ for OS and $56.63 \% \pm 5.47 \%$ for EFS) significantly lower than those reported in a European study (76.6\% and 68.3\%, respectively for OS and EFS) [13]. Furthermore, a Children's Oncology Group study in North America indicated that there were no significant deviations in survival or in incidence of high hazard ratios between Asian and Caucasian children with NB [14]. Although the sample size of Asian patients in this study was small it suggests that the poorer outcomes from NB in Vietnam are unlikely to be due to race differences. Other Southeast Asian countries have published biological data and research describing NB cohorts $[15,16]$. A study in Malaysia spanning 15 years, reported disease-free survival of $39 \%$ after a 2-year observation period [16]. In a Thailand cohort an OS of 52\% was reported after a 9-year follow up [15]. It may be implied that the outcome of NB in Vietnam was less favourable than Thailand but more favourable than Malaysia however, it is difficult to compare these studies due to the difference in time of follow-up and lack of updated information. In other Asian countries, for instance China, research showed that the EFS rate was 61\% [17] while in Japan, only $0.7 \%$ patients died within five years ( $99 \%$ for OS) [18]. Therefore, the survival rates of patients with NB in Vietnam were lower than those in Asian HIC such as Japan and China. However, all countries discussed in this study including Vietnam, report that survival rates of NB patients fall dramatically from diagnosis until three years postdiagnosis before demonstrating a plateau in survival rate [13-17].

In this cohort, most patients were diagnosed when they were older than 18 months of age (73.85\%) and at stage III and IV (73.85\%). In the aforementioned European study, between 1979-1989 most patients at diagnosis were also older than 18 months (62\%) and at stage III and IV [13]. However, patients diagnosed between 1997-2002 showed no significant differences in age groups or stage of NB [13]. It could be proposed that due to the advances in medical care within HICs, diagnosis has improved over time and NB can now be detected at earlier age and stages. However, the survival rate of patients was still impacted by the same variables, particularly that of NB stage which plays a significant role in prognosis of disease. Indeed, risk of OS and EFS of NB patients at stage III and IV $(13.54 \% \pm 3.56 \%$ and $31.21 \% \pm$ $7.03 \%$, respectively) was nearly 5.9 and 2.6 times higher significantly than those at stage I, II and IVs after 5-year follow up (Figure 2). Between 1997 - 2000 in the Europe cohort study, EFS of NB at stage III was about $73 \%$ and stage IV was around $35 \%$ (13). Comparatively, $66 \% \pm 16 \%$ NB patients in Thailand were stage III while $31 \% \pm 12 \%$ patients were stage IV after 5-year follow up [15]. Our reported OS and EFS rates in Vietnam were lower than these studies. Although age at diagnosis is a key factor that contributes to NB progression $[13,19]$ our study only reports a significant effect of age at diagnosis on prognosis of NB relapse but not for prognosis of NB outcome (Tables 1 and 2). Noticeably, increasing age is still a risk factor however it is dependent on MYCN oncogene status which also impacts the risk associated with stratification on other clinical features. The interaction between such factors must be further addressed to inform and optimise current guidelines on age stratification of NB cases in Vietnam.

The hallmark of MYCN amplification is well recognised as an important prognostic factor [18]. This study found that MYCN amplification accounted for $17.69 \%$ of the total cohort in Vietnam. The result is relatively consistent with previous studies in Europe and Thailand in that there was a strong association of $M Y C N$ amplification with poorer survival rate in NB $[13,15,20]$. MYCN-amplification in NB associated with decreased OS and EFS rates to $13.04 \% \pm 7.02 \%$ and $28.12 \% \pm 13.21 \%$, respectively after 5 -year observation; while the study in Europe found a higher percentage of survival rates $(72 \% \pm 7 \%$ for OS and $53 \% \pm 8 \%$ for EFS) [20]. In Thailand, children possessing $M Y C N$ amplification could not survive longer than 4 years of diagnosis with OS of $16 \% \pm 14 \%$ [15] which were slightly higher than our Vietnam NB cohort. In multivariate analysis, $M Y C N$ was independently associated with a decrease in OS and EFS; compatible with the findings of studies in Europe and Thailand $[13,20]$. Moreover, a poor outcome was dependent on $M Y C N$ - amplification when combined with age ( $>18$ months) or gender (male). Although survival time of male and female patients significantly differed by EFS $(P<0.05)$ (Supplementary Figure $\mathrm{S} 1)$, there was no evidence of an association between gender and the hazard ratio of NB patients (Table 3). Predictive value analysis revealed low sensitivity (23.3\%) of $M Y C N$-amplification status in predicting EFS (23.3\%) and high specificity of MYCN non-amplification in predicting EFS (85.1\%) (Table 2).

Treatment for high-risk, undifferentiated NB is a combination of intensive therapies including surgery, chemotherapy, radiotherapy, myeloablative therapy with autologous stem cell rescue, immunotherapy with FDA-approved anti-GD2 (disialoganglioside 2) and differentiation therapy with 13 -cis retinoic acid $[1,21]$. This multimodal therapy is not currently available for high-risk NB patients in Vietnam due to a lack of human resources, infrastructure and standard operative procedures and only $15-35 \%$ of expected cases in Vietnam could access a national pediatric-oncology programme) [22]. Aside from age, stage and MYCN status, NB risk groups can be further determined by analysis of chromosome $1 \mathrm{p}$, chromosome 11q and chromosome $17 \mathrm{q}$ copy number via multiplex ligation-dependent probe amplification (MLPA) [23] or comparative genomic hybridization (CGH) [24]. Anaplastic lymphoma kinase (ALK) gene amplification or mutation is also an optional test and, further, ALK inhibitors are available as a salvage treatment for refractory NB or following recurrence [25]. Recently, such tests have been used to guide therapy decisions [26]. Ongoing trials are also evaluating the use of rational combination of ${ }^{123} \mathrm{I}-\mathrm{MIBG}$, urine homovanilic acid/vanillylmandelic acid (HVA/VMA), MYCN, and DNA ploidy segmental chromosome abnormalities status, in an effort to provide more effective treatment. Unfortunately, such updated evidence-driven treatment in Vietnam and other LMICs is limited. Recently, the SIOP-PODC reported and adapted risk stratification and treatment guidelines considering the limited resources of diagnosis and treatment in LMICs [27]. Adopting such a strategy could be the key to preventing over-treatment while improving survival rates.

Overall, we provide the first report on clinicopathologic features of NB in Vietnam and describe prognostic factors associated with poor outcome. The results of this research could be applied to the quality management of diagnosis, prognosis and treatment in Vietnam and potentially other LMIC settings. Although there are some limitations in the retrospective design of our data collection, we highlight a major problem of decreased survival rates to NB in Vietnam. Additional research is necessary to further analyse the prognostic features of $\mathrm{NB}$ in Vietnam to inform optimal treatment strategies.

\section{Statement conflict of Interest}

The authors declare that there is no conflict of interest relating to this study. 


\section{Acknowledgements}

We thank Dr Michio YASUNAMI - Saga-Ken Medical Canter kosikan Life Science Institute for providing valuable feedback after reviewing this manuscript.

\section{Funding statement}

This work was supported by a grant from NAFOSTED - National Foundation for Science and Technology Development, Vietnam Ministry of Science (Grant Numbers: 106-YS.06-2014.48) and University of medicine and pharmacy at Ho Chi Minh City, Vietnam (Grant number: 2012-NCKH). The funders had no role in study design, data collection and analysis, decision to publish, or preparation of the manuscript.

\section{References}

1. Matthay KK, Maris MJ, Schleiermacher G, Nakagawara A, Mackall CL, et al. (2016) Neuroblastoma. Nat Rev Dis Primers 2: 16079. [Crossref]

2. Pizzo PA, Poplack DG (2015) Principles and practice of pediatric oncology: Lippincott Williams \& Wilkins.

3. Maris JM, Hogarty MD, Bagatell R, Cohn SL (2007) Neuroblastoma. Lancet 369: 2106-2120. [Crossref]

4. Smith MA, Seibel NL, Altekruse SF, Ries LA, Melbert DL, et al. (2010) Outcomes for children and adolescents with cancer: challenges for the twenty-first century. $J$ Clin Oncol 28: 2625-2634. [Crossref]

5. Gatta G, Botta L, Rossi S, Aareleid T, Bielska-Lasota M, et al. (2014) Childhood cancer survival in Europe 1999-2007: results of EUROCARE-5-a population-based study. Lancet Oncol 15: 35-47. [Crossref]

6. Magrath I, Steliarova-Foucher E, Epelman S, Ribeiro RC, Harif M, et al. (2013) Paediatric cancer in low-income and middle-income countries. Lancet Oncol 14: e104-e16. [Crossref]

7. Thiele CJ, Deutsch LA, Israel MA (1988) The expression of multiple protooncogenes is differentially regulated during retinoic acid induced maturation of human neuroblastoma cell lines. Oncogene 3: 281-288. [Crossref]

8. Beckers A, Van Peer G, Carter DR, Mets E, Althoff K, et al. (2015) MYCN-targeting miRNAs are predominantly downregulated during MYCN-driven neuroblastoma tumor formation. Oncotarget 6: 5204-5216. [Crossref]

9. Ambros PF, Ambros IM, Brodeur GM, Haber M, Khan J, et al. (2009) International consensus for neuroblastoma molecular diagnostics: report from the Internationa Neuroblastoma Risk Group (INRG) Biology Committee. Br J Cancer 100: 1471-1482. [Crossref]

10. De Preter K, Speleman F, Combaret V, Lunec J, Laureys G, et al. (2002) Quantification of MYCN, DDX1, and NAG Gene Copy Number in Neuroblastoma Using a Real-Time Quantitative PCR Assay. Mod Pathol 15: 159. [Crossref]

11. Iehara T, Hosoi H, Akazawa K, Matsumoto Y, Yamamoto K, et al. (2006) MYCN gene amplification is a powerful prognostic factor even in infantile neuroblastoma detected by mass screening. Br J Cancer 94: 1510-1515. [Crossref]

12. Wang M, Zhou C, Cai R, Li Y, Gong L (2013) Copy number gain of MYCN gene is a recurrent genetic aberration and favorable prognostic factor in Chinese pediatric neuroblastoma patients. Diagn Pathol 8: 5 [Crossref]
13. Moroz V, Machin D, Faldum A, Hero B, Iehara T, et al. (2011) Changes over three decades in outcome and the prognostic influence of age-at-diagnosis in young patients with neuroblastoma: a report from the International Neuroblastoma Risk Group Project. Eur J Cancer 47: 561-571. [Crossref]

14. Henderson TO, Bhatia S, Pinto N, London WB, McGrady P, et al. (2011) Racial and Ethnic Disparities in Risk and Survival in Children with Neuroblastoma: A Children's Oncology Group Study. J Clin Oncol 29: 76-82. [Crossref]

15. Shuangshoti S, Shuangshoti S, Nuchprayoon I, Kanjanapongkul S, Marrano P, et al (2012) Natural course of low risk neuroblastoma. Pediatr Blood Cancer 58: 690-694. [Crossref]

16. Ng SM, Abdullah WA, Lin HP, Chan LL (1999) Presenting features and treatment outcome of 78 Malaysian children with neuroblastoma. Southeast Asian J Trop Med Public Health 30: 149-153. [Crossref]

17. Yue ZX, Huang C, Gao C, Xing TY, Liu SG, et al. (2017) MYCN amplification predicts poor prognosis based on interphase fluorescence in situ hybridization analysis of bone marrow cells in bone marrow metastases of neuroblastoma. Cancer Cell Int 17: 43. [Crossref]

18. Iehara T, Hosoi H, Akazawa K, Matsumoto Y, Yamamoto K, et al. (2006) MYCN gene amplification is a powerful prognostic factor even in infantile neuroblastoma detected by mass screening. Br J Cancer 94: 1510-1515. [Crossref]

19. Brodeur GM, Bagatell R (2014) Mechanisms of neuroblastoma regression. Nat Rev Clin Oncol 11: 704-713. [Crossref]

20. Bagatell R, Beck-Popovic M, London WB, Zhang Y, Pearson AD, et al. (2009) Significance of MYCN amplification in international neuroblastoma staging system stage 1 and 2 neuroblastoma: a report from the International Neuroblastoma Risk Group database. Journal of Clinical Oncology. 27: 365-370. [Crossref]

21. DuBois SG, Kalika Y, Lukens JN, Brodeur GM, Seeger RC, et al. (1999) Metastatic sites in stage IV and IVS neuroblastoma correlate with age, tumor biology, and survival. J Pediatr Hematol Oncol 21: 181-9. [Crossref]

22. Ribeiro RC, Steliarova-Foucher E, Magrath I, Lemerle J, Eden T, et al. (2008) Baseline status of paediatric oncology care in ten low-income or mid-income countries receiving My Child Matters support: a descriptive study. Lancet Oncol 9: 721-729. [Crossref]

23. Tumer S, Altungoz O, Bagci O, Olgun HN (2016) The Detection of Genetic Parameters for Prognostic Stratification of Neuroblastoma Using Multiplex Ligation-Dependent Probe Amplification Technique. Genet Test Mol Biomarkers 20: 74-80. [Crossref]

24. Chan QR, Bilke S, Wei JS, Whiteford CC, Cenacchi N, et al. (2004) cDNA array-CGH profiling identifies genomic alterations specific to stage and MYCN-amplification in neuroblastoma. BMC Genomics 5: 70. [Crossref]

25. Debruyne DN, Bhatnagar N, Sharma B, Luther W, Moore NF, et al. (2016) ALK inhibitor resistance in ALKF1174L-driven neuroblastoma is associated with AXL activation and induction of EMT. Oncogene 35: 3681-91. [Crossref]

26. Pinto NR, Applebaum MA, Volchenboum SL, Matthay KK, London WB, et al. (2015) Advances in Risk Classification and Treatment Strategies for Neuroblastoma. J Clin Oncol 33: 3008-3017. [Crossref]

27. Parikh NS, Howard SC, Chantada G, Israels T, Khattab M, et al. (2015) SIOPPODC adapted risk stratification and treatment guidelines: Recommendations for neuroblastoma in low- and middle-income settings. Pediatr Blood Cancer 62: 13051316. [Crossref]

Copyright: (C2019 Bui C. This is an open-access article distributed under the terms of the Creative Commons Attribution License, which permits unrestricted use, distribution, and reproduction in any medium, provided the original author and source are credited. 